Methods A study was made cardiovascular disease of uygur population in hotan prefecture by registration in patients from Guma county hospital Total annual number of cardiovascular disease and coronary heart disease, Essential Hypertension, rheumatic heart disease, Cardiomyopathy, Pulmonary heart disease, Congenital heart disease and out comes were analysed.

Result The percentage of EH from 1996 13.7\% to 2005 22.4\%, the percentage of CHD from $19967.6 \%$ to $200514.2 \%$ the percentage of rheumatic heart disease from $19961.6 \%$ to $20053.5 \%$ the percentage of cardiomyopathy from 1996 1.9\% to 2005 3.3\% Pulmonary heart disease from 1996 4.4\% to 2005 5.7\%, the trend in cardiovascular diseases of Uighur population in hotan prefecture resent 10 years was change gradually, the annual percentage of Essential Hypertension, Coronary heart disease are rising significantly in resent 10 years, the annual percentage of rheumatic heart disease and Cardiomyopathy are slightly increased during this period (all $\mathrm{p}<0.05$ ).

Conclusion Prevention for cardiovascular disease should be strengthened in this area.

\section{Q0271 THE TREND OF CARDIOVASCULAR DISEASE OF UYGUR HOSPITALISED PATIENTS IN XINJIANG HETIAN. A SURVEY FROM 1996 TO 2005}

doi:10.1136/hrt.2010.208967.271

\begin{abstract}
${ }^{1}$ Yakefu Kalibinuer, ${ }^{2}$ Hasimu Buaijiaer, ${ }^{1}$ He Bingxian, ${ }^{2}$ Yuan Binbin. ${ }^{1}$ Heart Center, The First Hospital of Xinjiang Medical University; ${ }^{2}$ Department of Cardiology, Nanjing Benq Hospital, The Affiliated Hospital of Nanjing Medical University
\end{abstract}

Objective To investigate the trend of changes in cerebral- cardiovascular disease of Uygur hospitalised patients in Hetian from 1996 to 2005.

Methods Medical records of Uygur hospitalised patients from karakax county hospital and Gumar country hospital were investigated. Total annual number of cardiovascular disease and coronary heart disease (CHD), essential hypertension (EH), rheumatic heart disease, cardiomyopathy, pulmonary heart disease, congenital heart disease and out comes were analysed.

Result the percentage of EH from 1996 12.6\% to 2005 21\%, the percentage of CHD from $19968.8 \%$ to $200516.7 \%$ the percentage of rheumatic heart disease from $19961.3 \%$ to $20053.2 \%$ the percentage of cardiomyopathy from $19961.2 \%$ to $20052.3 \%$ the trend in cardiovascular diseases of Uygur population in Hetian prefecture resent 10 years vas change gradually, the annual percentage of $\mathrm{EH}, \mathrm{CHD}$ are rising significantly in resent ten years, the annual percentage of rheumatic heart disease and cardiomyopathy are slightly increased during this period (all $\mathrm{p}<0.05$ ) the annual percentage of pulmonary heart disease, congenital heart disease had no significantly change in resent ten years (all $\mathrm{p}>0.05$ ).

Conclusion the percentage of $\mathrm{EH}, \mathrm{CHD}$ are rising significantly in the Uygur population of this area. the percentage of rheumatic heart disease, Cardiomyopathy slightly increased during this period. Other Cardiovascular diseases had no significantly changes in resent ten years. Prevention for cardiovascular disease spatially CHD and $\mathrm{EH}$ should be strengthened in this area.

\section{e0272 LOW TESTOSTERONE LEVELS ARE INVERSELY ASSOCIATED WITH CAROTID ARTERY PLAQUE FORMATION}

doi:10.1136/hrt.2010.208967.272

Ma Qiang, Cheng Qingli, Wen Jing. Deptartment of Geriatric Nephrology General Hospital of Chinese Pla

Objective To study the relationship between endogenous sex hormone levels and atherosclerosis of the carotid artery measured by ultrasonography.
Methods Analysis of the healthy elders from a population-based cohort study in 9 communities of Beijing. carotid intima-media thickness (IMT) and atherosclerotic plaques were determined ultrasonographically. Sex hormone levels were measured by immunoassay. The data were analysed with ANOVA and logistic regression analysis.

Results There was a inverse association between testosterone and plaque formation in females $(p<0.001)$, whereas no association was found in males. Logistic regression analysis showed that females with a testosterone level in the lowest quintile $(<0.49 \mathrm{nmol} / \mathrm{l})$ were more likely $(\mathrm{OR}=3.11, \mathrm{p}=0.002)$ to be in the plaque formation independently of age and the other risk factors. Age $(\mathrm{OR}=1.07$ year1), $\mathrm{LDL}(\mathrm{OR}=1.65, \mathrm{p}=0.027)$, physical exercise $(\mathrm{OR}=0.54, \mathrm{p}=0.006)$, and IL-6 $(\mathrm{OR}=1.05, \mathrm{p}=0.022)$ were also independently associated with plaque formation.

Conclusions Testosterone concentrations are negatively associated with carotid artery atherosclerosis in females, experimental and prospective studies are needed to determine the possible therapeutic role of testosterone in atherosclerosis.

\section{e0273 RELATIONSHIP OF PERIPHERAL ARTERIAL DISEASE, CHRONIC KIDNEY DISEASE, AND MORTALITY IN CHINESE HYPERTENSIVE PATIENTS}

doi:10.1136/hrt.2010.208967.273

Li Jun, Luo Yingyi, Li Xiankai, Qiao Yongxia, Oin Liqiang, Hu Dayi, Li Jue. Heart Lung and Blood Vessel Center Tongji University School of Medicine Shanghai China

This study aimed to investigate the associations of chronic kidney disease (CKD), Peripheral arterial disease (PAD) and their combined effect with all-cause and Cardiovascular disease (CVD) mortality in Chinese hypertensive patients. Chinese ankle brachial index (ABI) cohort study including 2992 hypertensive patients aged 35 years or older were enrolled in 2004 and implemented the follow-up visit in 2007. 2505 participants completed baseline and follow-up data. Glomerular filtration rate (GFR) was estimated using the Modification of Diet in Renal Disease equation. ABI was calculated at baseline by measuring systolic pressures on bilateral brachial and tibial arteries. Mortality surveillance was completed from December 2007 to February 2008. Survival analysis was used to compare survival rate in different $\mathrm{CKD} / \mathrm{PAD}$ groups. The $\mathrm{RR}$ of death from all-cause and CVD were adjusted for potential confounders using a Cox regression model. The prevalence of PAD in hypertensive patients with and without CKD was $43.1 \%$ and $22.8 \%$, with significant difference between them $(p<0.001)$. For $\mathrm{CKD}$ and $\mathrm{PAD}$ group, $\mathrm{PAD}$ alone group, $\mathrm{CKD}$ alone group and neither CKD nor PAD group, the all-cause mortality was $38.6 \%$, $19.6 \%, 17.3 \%, 10.5 \%$, and CVD mortality was $23.3 \%, 13.7 \%$, $11.5 \%, 5.4 \%$, respectively, with significant differences among each other $(p<0.001)$. The survival rate was significantly lower in CKD and $\mathrm{PAD}$ group than in other three groups, for both all-cause and CVD mortality (log-rank: $p<0.001$ ). The patients in CKD and PAD group, PAD alone group and CKD alone group had the adjusted RRs of 2.966 (95\% CI 2.220 to 3.964), 1.523 (95\% CI 1.151 to 2.016 ) and 1.484 (95\% CI 1.067 to 2.063 ) for all-cause mortality and 3.402 (95\% CI 2.343 to 4.939 ), 2.024 (95\% CI 1.434 to 2.856 ), 1.843 (95\% CI 1.225 to 2.773 ) for CVD mortality, with no CKD or $\mathrm{PAD}$ group as the reference. Our study indicated that CKD is a risk factor for PAD. The hypertensive patients combined CKD and PAD had the highest risk for all-cause and CVD mortality. We suggest CKD patients should take ABI measurement for PAD early diagnosis and treatment, and GFR should be used to estimate renal function for $\mathrm{PAD}$ patients. 